\title{
High-Performance, Flexible Enzymatic Glucose Biosensor Based on ZnO Nanowires Supported on a Gold-Coated Polyester Substrate
}

\author{
Debabrata Pradhan, Farnaz Niroui, and K. T. Leung* \\ WATLab and Department of Chemistry, University of Waterloo, Waterloo, Ontario, Canada N2L 3G1
}

\begin{abstract}
The present work demonstrates the fabrication and performance of an enzymatic glucose biosensor based on ZnO nanowires (NWs) deposited on a Au-coated polyester (PET) substrate. Electrodeposition of ZnO NWs on the conducting PET substrate was carried out at $70^{\circ} \mathrm{C}$ in an aqueous electrolyte consisting of zinc nitrate mixed with potassium chloride. Glucose oxidase (GOx) was subsequently immobilized on the as-synthesized ZnO NWs, and the electrocatalytic properties of GOx-immobilized ZnO NWs were evaluated by amperometry. The resulting GOx/ZnO-NWs/Au/PET bioelectrode exhibits excellent electrocatalytic performance with a high sensitivity of $19.5 \mu \mathrm{A} \mathrm{mM}^{-1} \mathrm{~cm}^{-2}$, a low Michaelis-Menten constant of $1.57 \mathrm{mM}$, and a fast response time of $<5 \mathrm{~s}$ for the amperometric detection of glucose. The present study illustrates the feasibility of realizing light-weight, flexible, high-performance sensing devices using ZnO NWs.
\end{abstract}

KEYWORDS: glucose biosensor $\bullet$ zinc oxide $\bullet$ electrodeposition $\bullet$ plastic substrate $\bullet$ nanowires $\bullet$ electron microscopy

\section{INTRODUCTION}

$\mathrm{B}$ iosensors are important and essential devices in the present day-to-day clinical applications and environmental monitoring of pathogens and toxins $(1-3)$. In recent years, major developments have been made to increase the sensor performance by incorporating various nanostructured materials $(2,4-10)$. The inherently larger surface areas of nanomaterials offer a higher enzyme loading, which could dramatically improve the sensor activity. Furthermore, nanomaterials can be used to incorporate the activity of enzymes within its local nano-to-microscale environment, thereby enhancing the electron transfer between the active sites of the enzymes and the electrode (11). Of the several nanostructured materials that have been tested for biosensing applications, $\mathrm{ZnO}$ represents an excellent material with a high isoelectric point (of 9.5) that allows easy immobilization of an enzyme (with a low isoelectric point) through electrostatic interaction (12). Furthermore, its high chemical stability and non-toxic nature, as well as its biocompatibility and high electron transfer capability make ZnO a promising material for building an enzymatic sensor by immobilizing the appropriate biomolecules without electron mediators. Such type of ZnO-based biosensors can also be used to further develop implantable biosensing systems $(12-15)$.

There are currently well more than 220 million people living with diabetes world-wide, as per a recent report (November 2009) from the World Health Organization (16).

* Corresponding author. E-mail: tong@uwaterloo.ca.

Received for review May 11, 2010 and accepted July 12, 2010

DOI: 10.1021/am100413u

2010 American Chemical Society
Development of improved, low-cost glucose sensing devices will therefore have an enormous impact on a significant human population for future generations. In recent studies, $\mathrm{ZnO}$ or other nanomaterials have been deposited usually on a Au or Au-coated silicon or glass or other hard substrate, which was then used as a sensing device for glucose detection upon appropriate functionalization $(1,11,12)$. Devices based on plastic substrates represent the next technological challenges to reduce the weight and cost. In the present work, we demonstrate a working enzymatic glucose sensor based on $\mathrm{ZnO}$ nanowires (NWs) electrochemically deposited on a Au-coated polyester (PET) substrate. Glucose oxidase (GOx) has been immobilized on the ZnO NWs by simple physical adsorption, and the glucose sensing performance of the resulting GOx/ZnO-NWs/Au/PET bioelectrode has been characterized. The GOx/ZnO-NWs/Au/PET bioelectrode is found to be very sensitive to glucose, and it exhibits a very small Michaelis-Menten constant $(1.57 \mathrm{mM})$ and fast response $(<5 \mathrm{~s})$.

\section{EXPERIMENTAL DETAILS}

Figure 1 shows a schematic diagram of the bioelectrode fabricated in the present work. A flexible PET substrate $(1 \times 1$ $\mathrm{mm}^{2}, 0.2 \mathrm{~mm}$ thick) was first coated with a $2 \mathrm{~nm}$ thick Au layer by using a magnetron sputter-coater (Denton Desk II). The Aucoated PET substrate was then used as the working electrode for depositing ZnO NWs electrochemically in a conventional three-electrode cell with a $\mathrm{Ag} / \mathrm{AgCl}$ reference electrode and $\mathrm{a}$ $\mathrm{Pt}$ wire as the counter electrode. A CH Instruments 660A electrochemical workstation was employed for the electrodeposition of ZnO NWs by amperometry at a constant applied potential of $-0.6 \mathrm{~V}$ (with reference to the $\mathrm{Ag} / \mathrm{AgCl}$ electrode) at $70{ }^{\circ} \mathrm{C}$ in an electrolyte of $1 \mathrm{mM} \mathrm{Zn}\left(\mathrm{NO}_{3}\right)_{2} \cdot 6 \mathrm{H}_{2} \mathrm{O}$ mixed with $100 \mathrm{mM} \mathrm{KCl}$ supporting electrolyte. The as-grown ZnO NW film was characterized by field emission scanning electron micros- 


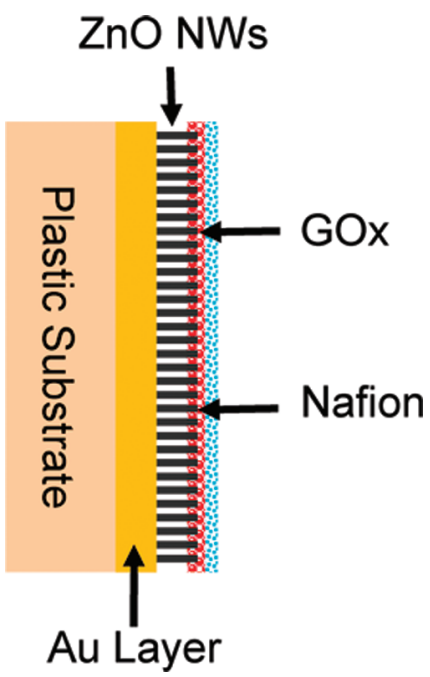

FIGURE 1. Schematic diagram of GOx/ZnO-NWs/Au/PET bioelectrode used in the present study for glucose monitoring. The Au-coated PET substrate is deposited with $\mathrm{ZnO}$ nanowires (NWs) immobilized with glucose oxidase (GOx) in a phosphate buffer saline solution mixed with Nafion.

copy (SEM), transmission electron microscopy (TEM), and glancing-incidence X-ray diffraction (GIXRD). The $\mathrm{ZnO}-\mathrm{NW} / \mathrm{Au} /$ PET sample was then dipped in a solution of GOx (Fluka, $1 \mathrm{mg} /$ $\mathrm{mL}$ ) prepared in a $50 \mathrm{mM}$ phosphate buffer saline (PBS) solution mixed with Nafion (5\%) and left soaking overnight to allow GOx immobilization to take place. Nafion is known to be used as a covering network, which provides a biocompatible environment to the enzyme without interfering with the biosensor $(17,18)$. Subsequently, the sample was washed thoroughly several times with the PBS solution to remove excess GOx. The glucose sensing properties of the resulting GOx/ZnO-NWs/Au/PET bioelectrode were studied by an amperometric method, in which the amperometric response was measured upon successive increases in glucose concentration (at steps of $0.020,0.050$, or $0.100 \mathrm{mM}$ ) in the PBS solution under constant stirring.

\section{RESULTS AND DISCUSSION}

Figure 2 a shows the SEM image of ZnO NWs electrodeposited on the Au-coated PET substrate at $70{ }^{\circ} \mathrm{C}$ in an electrolyte of $1 \mathrm{mM} \mathrm{Zn}\left(\mathrm{NO}_{3}\right)_{2} \cdot 6 \mathrm{H}_{2} \mathrm{O}$ mixed with $100 \mathrm{mM}$ $\mathrm{KCl}$. The ZnO NWs appear to grow near-perpendicularly to the Au-coated PET substrate with a uniform distribution over the substrate. The cross-sections of the Zn NWs also appear to be homogeneous with an average diameter of $70-80 \mathrm{~nm}$ (and a small size distribution). The corresponding average length of these NWs is measured to be $\sim 300 \mathrm{~nm}$ from the low-magnification TEM image shown in Figure 2b (left inset). The high-resolution TEM image (Figure 2b) clearly shows a two-dimensional lattice pattern with an interlayer spacing of $2.6 \AA$, indicating the highly crystalline nature of the electrodeposited $\mathrm{ZnO}$ NWs and their $c$-axis growth along the (0001) plane. The corresponding selected area electron diffraction shows the spot diffraction pattern (Figure $2 \mathrm{~b}$, right inset) depicting the single-crystalline nature and [0001] growth direction of the ZnO NW $(19,20)$.

Figure 3 shows the corresponding GIXRD pattern of $\mathrm{ZnO}$ NWs electrodeposited on the Au-coated PET substrate. The positions of the observed major XRD features (Figure 3a) are well matched to those of the reference wurtzite structure of ZnO (JDPDS 01-070-2551) (21) shown in Figure 3b. The

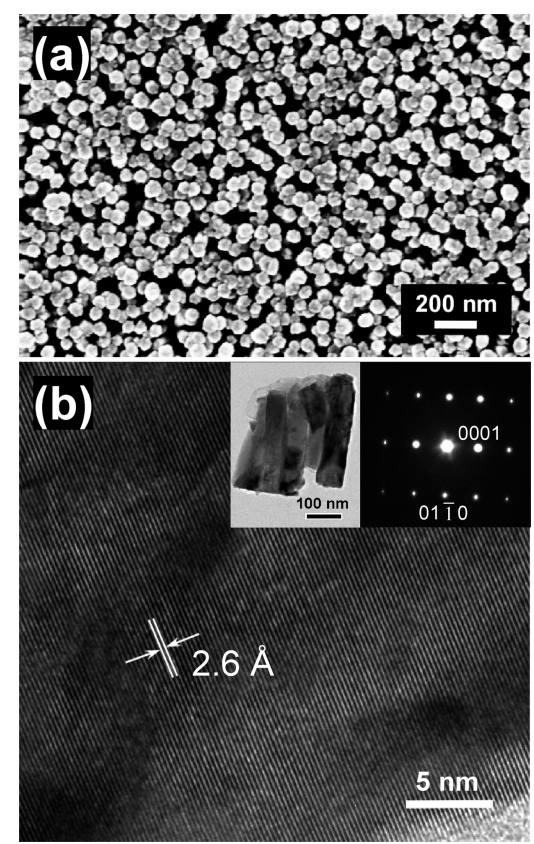

FIGURE 2. (a) SEM image and (b) high-resolution TEM image of $\mathrm{ZnO}$ nanowires electrodeposited on a Au-coated PET substrate at $70^{\circ} \mathrm{C}$. The insets show the corresponding low-magnification TEM image (left) and selected area electron diffraction pattern (right).

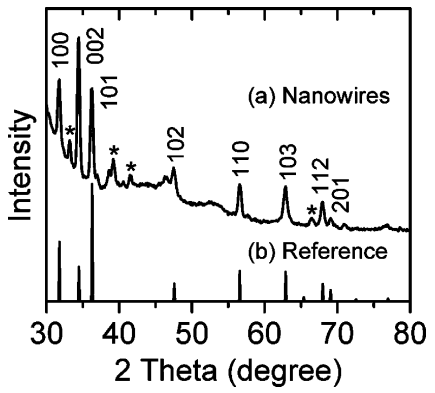

FIGURE 3. Glancing-incidence XRD spectra of (a) $\mathrm{ZnO}$ nanowires electrodeposited on Au-coated PET substrate at $70^{\circ} \mathrm{C}$. The crystallographic identifications of the $\mathrm{ZnO}$ peaks are labeled in accord with (b) the reference spectrum for ZnO (JCPDS 01-070-2551). Features arising from the Au-coated PET substrate are marked by asterisks.

weaker XRD features marked with asterisks belong to the underlying Au-coated PET substrate. The strongest peak located at $34.45^{\circ}$ is attributed to the (002) plane of $\mathrm{ZnO}$, in good accord with the well-known observation that onedimensional $\mathrm{ZnO}$ nanostructures (including nanowires and nanorods) prefer to grow along the $c$-axis, i.e., in the [002] direction (normal to the substrate) (22).

The efficiency of the GOx/ZnO-NWs/Au/PET bioelectrode is evaluated by an amperometric method, and the corresponding performance data were compared with other nanomaterial-based glucose sensors reported in the literature in Table 1 . Figure 4 shows a typical steady-state amperometric response of the GOx/ZnO-NWs/Au/PET bioelectrode on successive increase in glucose concentrations with different steps $(0.02,0.05$, and $0.1 \mathrm{mM})$ in a $1 \mathrm{mM}$ PBS solution ( $\mathrm{pH} 7.4$ ), at an applied potential of $0.8 \mathrm{~V}$. During the electrochemical process, the GOx enzyme immobilized on the $\mathrm{ZnO}$ NWs catalyses the oxidation reaction of glucose to gluconolactone and hydrogen peroxide. Subsequently, hydrogen peroxide is oxidized causing an increase in current 
Table 1. Comparison of the Amperometric Glucose Biosensors Based on Various Glucose-Oxidase-Modified Nanomaterials on Different Substrates

\begin{tabular}{|c|c|c|c|c|c|}
\hline working electrode & $\begin{array}{c}\text { sensitivity } \\
\left(\mu \mathrm{A} \mathrm{mM}^{-1} \mathrm{~cm}^{-2}\right)\end{array}$ & $\begin{array}{c}\text { Michaelis-Menten } \\
\text { constant (mM) }\end{array}$ & $\begin{array}{l}\text { detection limit } \\
(\mu \mathrm{M})\end{array}$ & $\begin{array}{l}\text { response } \\
\text { time (s) }\end{array}$ & reference \\
\hline ZnO NWs on Au-coated PET & 19.5 & 1.57 & $<50$ & $<5$ & this work \\
\hline $\mathrm{ZnO}$ nanocombs on $\mathrm{Au}$ & 15.33 & 2.19 & 20 & $<10$ & $(12)$ \\
\hline carbon-decorated ZnO NWs on Ti & 35.3 & 1.54 & 1.0 & 5 & (23) \\
\hline ZnO nanonails on $\mathrm{Au}$ & 24.61 & 14.7 & 5.0 & $<10$ & (11) \\
\hline $\mathrm{CeO}_{2}$ on Au-coated glass & 0.00287 & 0.01355 & 12.0 & $<5$ & $(4)$ \\
\hline CdS nanoparticles on graphite & 7.0 & 5.1 & 0.05 & N/A & $(25)$ \\
\hline
\end{tabular}

[via the reaction $\mathrm{H}_{2} \mathrm{O}_{2} \rightarrow 2 \mathrm{H}^{+}+\mathrm{O}_{2}+2 \mathrm{e}^{-}$]. Evidently, the successive increase in glucose concentration at $0.02 \mathrm{mM}$ increments induces negligible change in the oxidation current, while a discernible increase in the response current is observed with the increase in glucose concentration at 0.05 $\mathrm{mM}$ increments (Figure 4). On the other hand, a significant increase in the response current is found with the increase in glucose concentration at $0.1 \mathrm{mM}$ increments. The time needed to reach a steady-state current upon addition of glucose is found to be less than $5 \mathrm{~s}$, which indicates a good electrocatalytic performance and a fast electron exchange response of the GOx/ZnO-NWs bioelectrode. The detection limit for the GOx/ZnO-NWs/Au/PET bioelectrode is found to be $0.05 \mathrm{mM}$ based on a signal-to-noise ratio of 5 .

To evaluate the useful working detection range for the GOx/ZnO-NWs/Au/PET bioelectrode, we repeat the amperometric response measurement for a more extended set of glucose concentrations. Figure $5 \mathrm{a}$ shows the calibration curve that clearly exhibits a linear dependence of the response current on the glucose concentration at low concentration over a larger range, $0.2-2.0 \mathrm{mM}$. With increasing glucose concentration above $2.0 \mathrm{mM}$, the response current appears to reach a saturated value (below $6 \mu \mathrm{A}$ ) because of the saturation of enzyme active sites at a higher glucose concentration. From the slope of the linear portion of the calibration curve and the working area of bioelectrode, a very high sensitivity of $19.5 \mu \mathrm{A} \mathrm{mM}^{-1} \mathrm{~cm}^{-2}$ is calculated for the GOx/ZnO-NWs/Au/PET bioelectrode. Indeed, this value is found to be higher than many of other nanomaterial-based

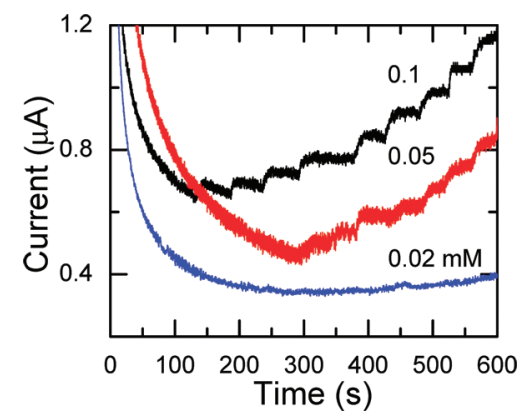

FIGURE 4. Amperometric response of GOx/ZnO-NWs/Au/PET bioelectrode with successive increase in glucose concentration at different increments $(0.02,0.05$, and $0.1 \mathrm{mM})$ in a $1 \mathrm{mM}$ PBS buffer solution at an applied potential of $0.8 \mathrm{~V}$ (vs. $\mathrm{Ag} / \mathrm{AgCl}, 3 \mathrm{M} \mathrm{KCl}$ reference electrode). biosensors reported previously (Table 1). The high sensitivity obtained in the present work indicates the excellent affinity for GOx immobilization, high electrocatalytic activity, and good biocompatibility of electrochemically synthesized ZnO NWs. The apparent Michaelis-Menten constant $\left(K_{\mathrm{m}}^{\mathrm{app}}\right)$, which is a measure of the enzyme-substrate kinetics of the glucose biosensor, can be calculated from the Lineweaver-Burk equation, i.e., $1 / V=\left(K_{m}^{\mathrm{app}} / V_{\max }\right)(1 / S)+1 / V_{\max }$, where $V_{\max }$ is the maximum reaction velocity obtained from the Lineweaver-Burk plot and $\mathrm{S}$ is the surface concentration. The $K_{m}^{\text {app }}$ value calculated from the Lineweaver-Burk plot (Figure $5 \mathrm{~b}$ ) for the GOx/ZnO-NWs/Au/PET bioelectrode is $1.57 \mathrm{mM}$, which is considerably smaller than most of the reported nanomaterial-based enzymatic glucose sensors (Table 1), again confirming the excellent GOx enzyme affinity on the ZnO NWs. This is likely due to the biocompatible nature and larger surface area of the ZnO NWs that provide a suitable microenvironment for higher GOx loading. Furthermore, the positively charged ZnO NWs tend to bind strongly with the negatively charged GOx through electrostatic attraction. We illustrate in the present work that these favorable properties
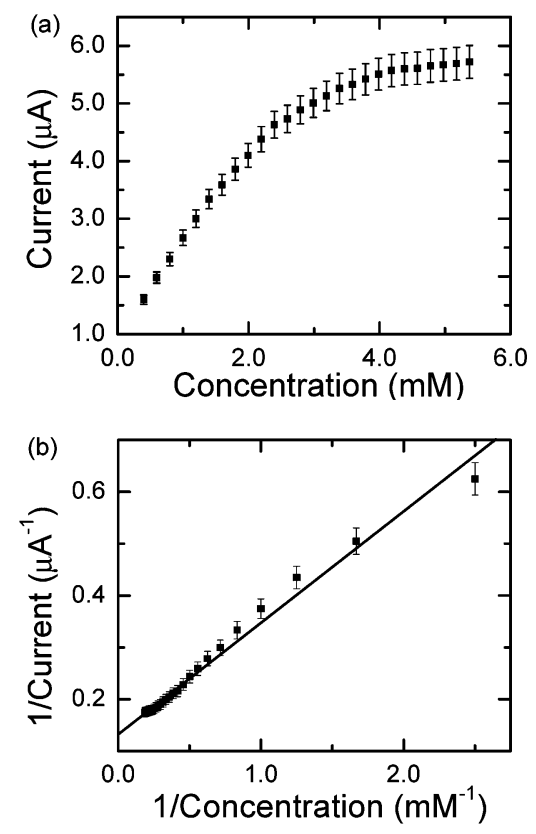

FIGURE 5. (a) Calibration curve, and (b) the corresponding Lineweaver-Burk plot of GOx/ZnO-NWs/Au/PET bioelectrode over an extended concentration range. 
of ZnO NWs can be harnessed for biosensor applications. The present sensor is found to be quite stable and only a slight decrease in the activity (less than $5 \%$ ) is observed after 7 days. Unlike most of the previous studies where $\mathrm{Au}$ substrate is used for fabricating bioelectrodes for glucosesensing application (Table 1), the use of Au-coated PET substrate offers important commercial advantages of lower cost and improved portability of light-weight and flexibility.

\section{SUMMARY}

In the present work, we demonstrate the performance of a light-weight, flexible glucose-sensing bioelectrode based on ZnO NWs electrochemically deposited on a Au-coated PET substrate. Direct electrodeposition offers a simple, onestep procedure to produce uniformly distributed, vertically oriented $\mathrm{ZnO}$ NWs on a flexible Au-coated PET substrate. Using an amperometric method, the GOx/ZnO-NWs/Au/PET bioelectrode is found to exhibit excellent and fast glucosesensing performance, with a high sensitivity of $19.5 \mu \mathrm{A}$ $\mathrm{mM}^{-1} \mathrm{~cm}^{-2}$ and a low Michaelis-Menten constant of 1.57 $\mathrm{mM}$, making $\mathrm{ZnO} \mathrm{NW}$-based biosensors promising candidates for future commercialization.

Acknowledgment. This work was supported by the Natural Sciences and Engineering Research Council of Canada.

\section{REFERENCES AND NOTES}

(1) Singh, S. P.; Arya, S. K.; Pandey, P.; Malhotra, B. D.; Saha, S.; Sreenivas, K.; Gupta, V. Appl. Phys. Lett. 2007, 91, 063901.

(2) Rout, C. S.; Govindaraj, A.; Rao, C. N. R. J. Mater. Chem. 2006 16, 3936.

(3) Lao, C. S.; Kuang, Q.; Wang, Z. L.; Park, M.-C.; Deng, Y. Appl Phys. Lett. 2007, 90, 262107.
(4) Ansari, A. A.; Solanki, P. R.; Malhotra, B. D. Appl. Phys. Lett. 2008, 92, 263901

(5) Wang, X.; Chen, Y.; Gibney, K. A.; Erramilli, S.; Mohanty, P. Appl. Phys. Lett. 2008, 92, 013903.

(6) Hrapovic, S.; Liu, Y.; Male, K. B.; Luong, J. H. T. Anal. Chem. 2004, 76, 1083.

(7) Lin, Y.; Lu, F.; Tu, Y.; Ren, Z. Nano Lett. 2004, 4, 191.

(8) Zhang, X.; Wang, H.; Bourgeois, L.; Pan, R.; Zhao, D.; Webley, P. A. J. Mater. Chem. 2008, 18, 463.

(9) Zhang, X.; Wang, G.; Zhang, W.; Hu, N.; Wu, H.; Fang, B. J. Phys. Chem. C 2008, 112, 8856

(10) Chen, W.; Yao, H.; Tzang, C. H.; Zhu, J.; Yang, M.; Lee, S.-T. Appl. Phys. Lett. 2006, 88, 213104

(11) Umar, A.; Rahman, M. M.; Kim, S. H.; Hahn, Y. B. J. Nanosci. Nanotechnol. 2008, 8, 3216.

(12) Wang, J. X.; Sun, X. W.; Wei, A.; Lei, Y.; Cai, X. P.; Li, C. M.; Dong, Z. L. Appl. Phys. Lett. 2006, 88, 233106.

(13) Kumar, S.; Gupta, V.; Sreenivas, K. Nanotechnology 2006, 16, 1167.

(14) Krishnamoorthy, S.; Bei, T.; Zoumakis, E.; Chrousos, G. P.; Iliadis, A. A. Biosens. Bioelectron. 2006, 22, 707.

(15) Wei, A.; Sun, X. W.; Wang, J. X.; Lei, Y.; Cai, X. P.; Li, C. M.; Dong, Z. L.; Huang, W. Appl. Phys. Lett. 2006, 89, 123902.

(16) http://www.who.int/mediacentre/factsheets/fs312/en/index.html.

(17) Yang, Y.; Yang, H.; Yang, M.; Liu, Y.; Shen, G.; Yu, R. Anal. Chim. Acta 2004, 525, 213.

(18) Xu, J.-J.; Yu, Z.-H.; Chen, H.-Y. Anal. Chim. Acta 2003, 463, 239.

(19) Ku, C.-H.; Yang, H.-H.; Chen, G.-R.; Wu, J.-J. Cryst. Growth Des. 2008, 8, 283.

(20) Bae, S. Y.; Seo, H. W.; Park, J. J. Phys. Chem. B 2004, 108, 5206.

(21) JCPDS file reference number 01-076-0704. PDF-2 Database; International Center for Diffraction Data: Newtown Square, PA, 2004.

(22) Wu, J.-J.; Liu, S.-C. Adv. Mater. 2002, 14, 215.

(23) Liu, J.; Guo, C.; Li, C. M.; Li, Y.; Chi, Q.; Huang, X.; Liao, L.; Yu, T. Electrochemistry Commun. 2009, 11, 202.

(24) Zhao, Z. W.; Chen, X. J.; Tay, B. K.; Chen, J. S.; Han, Z. J.; Khor, K. A. Biosens. Bioelectron. 2007, 23, 135.

(25) Huang, Y.; Zhang, W.; Xiao, H.; Li, G. Biosens. Bioelectron. 2005, 21,817

AM100413U 